\title{
Analysis of calcium carbonate for differentiating between pigments using terahertz spectroscopy
}

\author{
Maya Mizuno \\ mmizuno@nict.go.jp \\ Kaori Fukunaga \\ Shingo Saito \\ Iwao Hosako
}

\author{
National Institute of Information and Communications Technology, Tokyo, Japan \\ National Institute of Information and Communications Technology, Tokyo, Japan \\ National Institute of Information and Communications Technology, Kobe, Japan \\ National Institute of Information and Communications Technology, Tokyo, Japan
}

\begin{abstract}
Calcium carbonate that is used as an art pigment exhibits strong absorption at approximately $3 \mathrm{THz}$. In this study, the authors investigated the relation between the absorption and the condition of calcium carbonate crystals. By employing terahertz time-domain spectrometer (0.5-4 THz), they verified that terahertz absorption energy depended on the crystal direction and crystal shape of the powder sample due to large birefringence. Further, the authors observed the difference in the crystal structure (calcite or aragonite) and the presence of impurities in natural calcium carbonate such as shells through terahertz absorbance spectra. The absorbance peak value of calcite at around $3 \mathrm{THz}$ was four times as large as the peak value of aragonite. The absorbance spectral width increased because of the presence of these impurities. From the above observation, this study demonstrated that a certain kind of calcium carbonate crystal could be distinguished by terahertz spectroscopy. [DOI: 10.2971/jeos.2009.09044]
\end{abstract}

Keywords: terahertz spectroscopy, calcite, aragonite, carbonate group

\section{INTRODUCTION}

For art conservation and restoration of cultural properties, scientific investigation for determining the original raw materials of a pigment is important in deciding the raw materials that should be used for the restoration. Calcium carbonate $\left(\mathrm{CaCO}_{3}\right)$ has been used as pigments and wall painting bases since ancient times. Therefore, it is important to determine the raw materials used as a source for $\mathrm{CaCO}_{3}$ as a result of the aged deterioration of cultural properties. Shell and limestone are commonly used as raw materials for $\mathrm{CaCO}_{3}$. At the present time, the kind of material is differentiated by employing analysis apparatuses such as an X-ray fluorescence spectrometer, X-ray diffractometer and FT-IR spectrometer [1]. Xray fluorescence can be used to search atoms and compounds, and X-ray diffraction can be used to verify the crystal structure of the raw material. FT-IR spectroscopy in the range of mid-infrared can also distinguish between crystal polymorphs such as calcite and aragonite by comparing the different spectral pattern; furthermore, FT-IR spectroscopy has the possibility of being able to obtain the information on composites (glue, organic matter, etc.) included in the pigment [2]. In the case of shells, a few organic materials, which combine with inorganic matter, control the shells' crystal polymorphism and size [3]. The identification of the combined state of organic matter and inorganic matter possibly helps in differentiating between the shells. However, in the range of mid-infrared, where we can observe molecular vibration having a relatively high energy, it is difficult to observe a weak interaction between molecules, such as that due to a hydrogen bond and van der Waals force. Therefore, we employed a terahertz spectrometer [4]-[6] that could observe low energy vibration, and could verify the possibility of observing the combined state with impurities and of sensitively differentiating between pigments.

In this study, we measured the absorption spectra of a single crystal (natural calcite), high purity $\mathrm{CaCO}_{3}$ powder, and shell powder at $0.5-4 \mathrm{THz}$, where the scattering effect of the powder sample was small. By employing terahertz time-domain spectroscopy (THz-TDS), we verified that the terahertz spectral pattern depended on the crystal direction, crystal structure and the presence of impurities. From the above observation, this study indicated the possibility that the raw materials of a white pigment based on $\mathrm{CaCO}_{3}$ could be distinguished by terahertz spectroscopy.

\section{TERAHERTZ SPECTROSCOPY OF CALCIUM CARBONATE}

\subsection{Single crystal measurement}

We used a THz-TDS system developed by Tochigi Nikon Corporation to measure the absorbance of $\mathrm{CaCO}_{3}$. The frequency resolution was set to $0.059 \mathrm{THz}$, the pressure in the terahertz system was less than $20 \mathrm{~Pa}$, and the temperature was approximately $28^{\circ} \mathrm{C}$. First, we analyzed a single crystal of natural calcite (from Mexico) to understand the relation between 


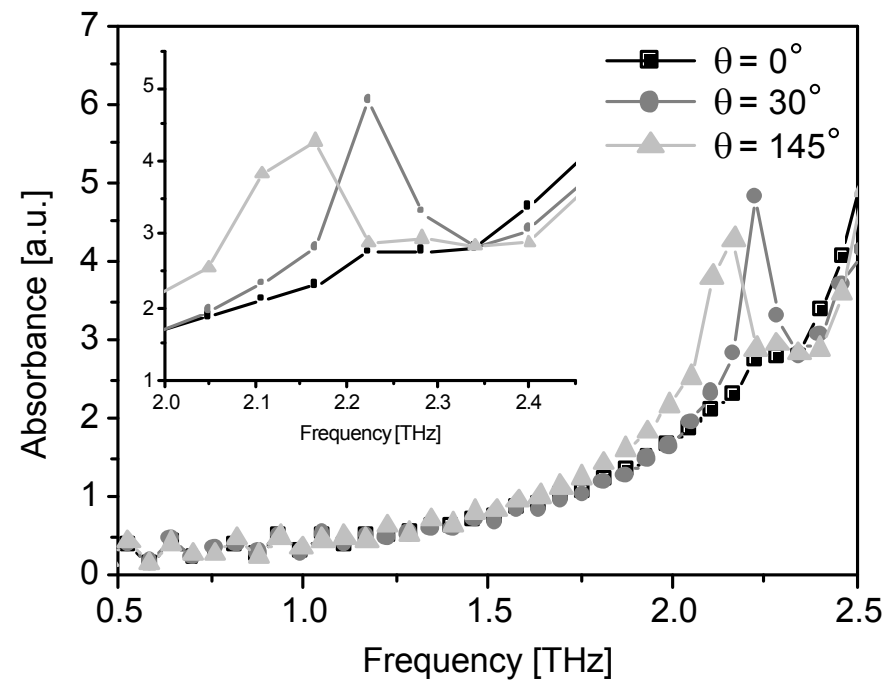

FIC. 1 Terahertz absorbance spectra of natural calcite crystal at $0^{\circ}, 30^{\circ}$ and $145^{\circ}$, and the magnified view of the absorption peaks.

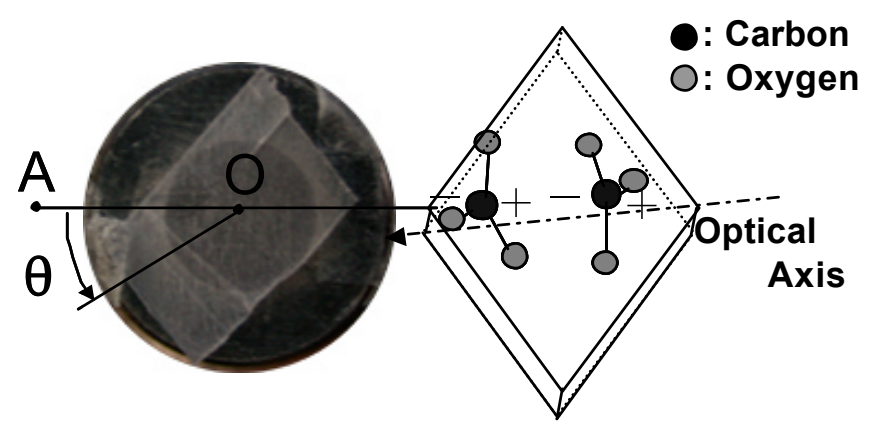

FIG. 2 Photography of the natural calcite crystal, definition of the angle $\theta$ (left), and the relative arrangement of atoms within calcite as seen at the (104) plane (right). The dashed arrow shows optical axis.

absorbance and crystal direction. The calcite cut in the (104) cleavage plane, which had simple charge distribution, was ground to a thickness of approximately $0.38 \mathrm{~mm}$, and was placed on a rotation stage to measure the angle dependence of absorbance. Figure 1 shows the absorbance spectra of the natural calcite at $\theta=0^{\circ}, 45^{\circ}$ and $135^{\circ}$ using the focus optical system. Here, $\theta$ was defined as the angle between the terahertz electric field direction and the bisector of obtuse angles between face edges in the calcite $\left(\sim 102^{\circ}\right)$, and this $\theta$ is shown by a solid arrow in Figure 2. We verified that the absorption was strong at a high frequency due to the vibration of calcite around $3 \mathrm{THz}[7,8]$, and we could not observe the spectra of the natural calcite above $2.5 \mathrm{THz}$ using the THz-TDS (S/N: $\sim 10 \mathrm{~dB}$ at $4 \mathrm{THz}$ ). When $\theta$ was $30^{\circ}$ and $145^{\circ}$, we could observe an absorption peak at around 2.2 THz. To speculate these absorption causes, we analyzed the angle dependence of absorbance at 2.12, 2.23 and $2.4 \mathrm{THz}$. The angle dependence of the absorption around $3 \mathrm{THz}$ was observed at $2.4 \mathrm{THz}$. The absorbance from 0 to $360^{\circ}$ is shown in Figure 3 . We could verify that the absorbance at 2.12, 2.23 and $2.4 \mathrm{THz}$ were maximum at angles of $145^{\circ}, 30^{\circ}$ and $0^{\circ}$, respectively, and this property on the same crystal exhibited reproducibility. Generally, the carbonate group in calcite crystal is arranged as shown in Figure 2 [9]. Therefore, the dipole of the carbonate group is arranged on the optical axis (axis of anisotropy), and that

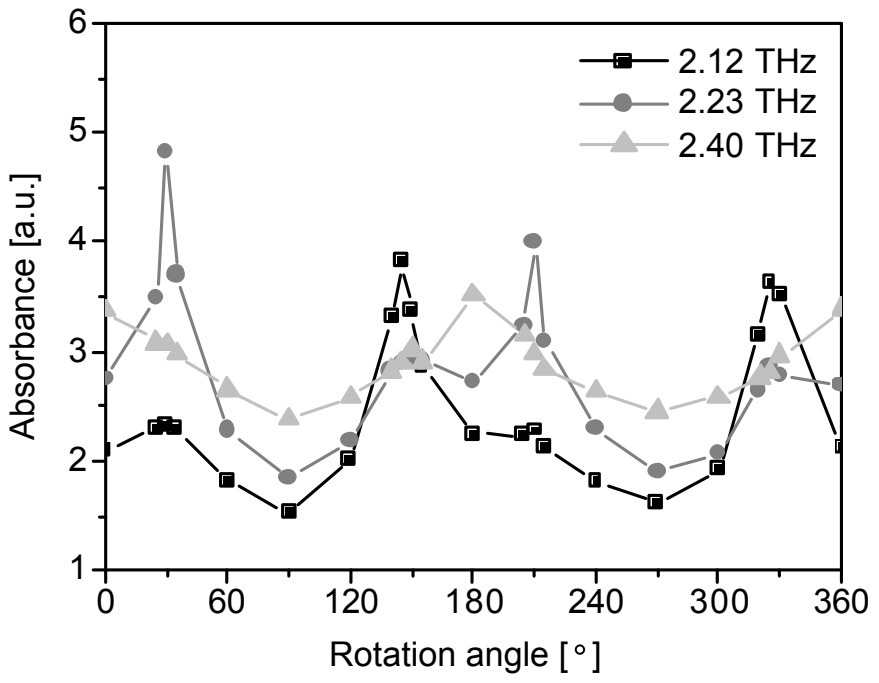

FIG. 3 Terahertz absorbance variation with $\theta$ at $2.12,2.23$ and $2.40 \mathrm{THz}$.

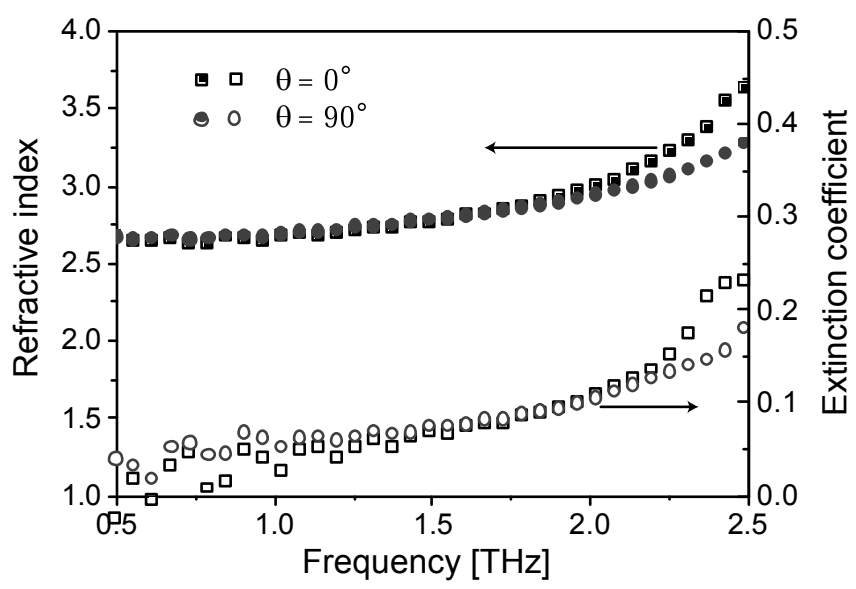

FIC. 4 Refractive index (filled symbols) and extinction coefficient (unfilled symbols) at $0^{\circ}$ (square) and $90^{\circ}$ (circle).

is the reason for the large birefringence (angle dependence of refractive index). The refractive index of an extraordinary ray in calcite is smaller than that of an ordinary ray (negative crystal). However, the relation of the refractive index was reversed at around $2.4 \mathrm{THz}$ (see Figure 4). Further, the extinction coefficient of the extraordinary ray was larger than that of the ordinary ray. By these differences between the ordinary ray and the extraordinary ray, when a terahertz wave is incident vertically on the (104) plane, the wave is elliptically polarized when it passes through the crystal. This polarization is not parallel to the incident terahertz wave polarization at $\theta \neq 0^{\circ}$ and $90^{\circ}$. Therefore, the graph of Figure 4 includes the angle dependence of detector efficiency on a photoconductive antenna (dipole antenna). However, we could observe the absorption property in each crystal direction in Figure 4. Thus, we could estimate the detection loss at the photoconductive antenna, which depended on the polarization, was less than $15 \mathrm{~dB}$. The detection loss was calculated from the difference of absorbance between $\theta=0^{\circ}$ and $90^{\circ}$.

From the above angle dependence of the absorbance, we analyzed the molecules related to absorption. In the study of terahertz spectroscopy, when the terahertz electric field is parallel to a dipole, the absorption of the dipole vibration be- 
comes large due to the variation in dipole polarization [10]. From this method, we realized that the absorption at around $2.4 \mathrm{THz}$, which became strong when $\theta$ was $0^{\circ}$, was affected by the dipole vibration of the carbonate group. However, we observed that the extinction coefficient becomes large at around $2.4 \mathrm{THz}$ also when $\theta$ becomes $90^{\circ}$, as shown in Figure 4. Thus, we conjecture that absorption due to an out-of-plane vibration and an in-plane vibration of the carbonate group can be observed at around $3 \mathrm{THz}$. These vibrations corresponding to the transverse optic mode have been investigated in earlier studies $[7,8]$. The absorption peak of the out-of-plane vibration is observed at lower frequency than that of the in-plane vibration. In the case of absorption at $30^{\circ}$ and $145^{\circ}\left(-35^{\circ}\right)$, we speculate that the absorption has a relation with the vibration of $\mathrm{C}-\mathrm{O}$ in the carbonate group because the $\mathrm{C}-\mathrm{O}$ charge distribution on the (104) plane is axisymmetric as $\theta=0^{\circ}$ [11]. It has the possibility of selectively inducing the $\mathrm{C}-\mathrm{O}$ vibration when the terahertz electric field is nearly parallel to $\mathrm{C}-\mathrm{O}$, even though it was difficult to distinguish an absorption peak of $\mathrm{C}$ $\mathrm{O}$ vibration and the absorption property of an ordinary ray at $90^{\circ}$, where there is $\mathrm{C}-\mathrm{O}$. We conjecture that this vibration is in the longitudinal optic mode that occurs because the impurities are combined with C-O instead of $\mathrm{Ca}$ in natural calcite [12]. From the above analysis, we verified that we can observe various absorption peaks of carbonate groups at around $3 \mathrm{THz}$.

\subsection{Measurement of calcite powder}

Before the measurements of pigments, we also checked if the powder samples have the same fundamental properties. We

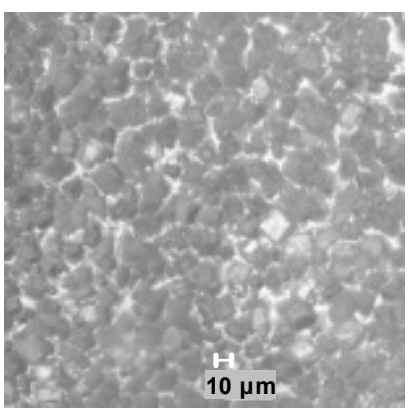

(a)

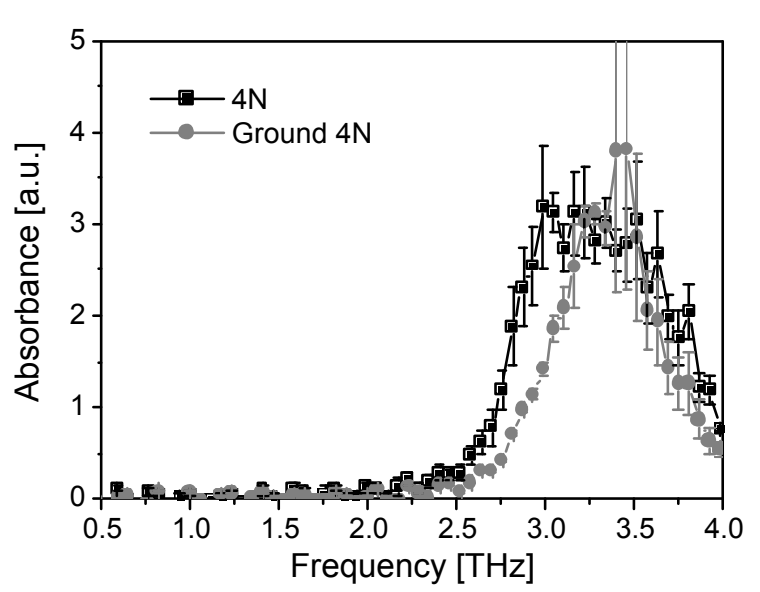

(c)

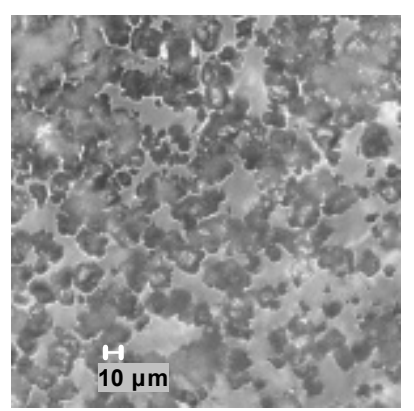

(b) used a $99.99 \%$ purity powder (4N, Kanto Chemical Co., Inc.) and ground $4 \mathrm{~N}$ powder to verify the relation between absorbance and crystal shape. The powder sample with a mass of approximately $7 \mathrm{mg}$ was placed in a spacer (hole diameter: $10 \mathrm{~mm}$, thickness: $0.1 \mathrm{~mm}$ ) on a synthetic quartz plate. Figures 5(a) and 5(b) are images of powder samples acquired by a digital microscope (KEYENCE, VHX-900) at a magnification of 1000 times. Figure 5(a) shows that $4 \mathrm{~N}$ consists of a calcite crystal of approximately $10 \mu \mathrm{m}$. In Figure 5(b), we verified that the crystal size of ground $4 \mathrm{~N}$ powder was less than that of $4 \mathrm{~N}$ powder; further, the shape was not rhombohedral due to grinding. Figure 5(c) shows the absorbance spectra of these powder samples. The errors bars show the errors in the measurement of absorption, which depend on the differences in the thicknesses and mass of the three samples. At above $3 \mathrm{THz}$, The errors especially depend on the S/N of the TDS system. The figure shows that we cannot observe absorption peaks at 2.12 and $2.23 \mathrm{THz}$ in case of the powder sample. The samples have an absorption peak only at approximately $3 \mathrm{THz}$. Here, there is no angle dependence of spectra because crystal directions are random. From these results, we conjecture that the absorption of $\mathrm{C}-\mathrm{O}$ at the low frequency side can be observed only when the sample is a natural crystal arranged in the crystal direction. Moreover, we verified that the spectral width of ground $4 \mathrm{~N}$ powder was narrower than 4 N's width. We speculate that the width decreased because the broadband absorption of the extraordinary ray decreased due to the particle shape effect and the decrease in particle size [13].

\section{DIFFERENTIATING BETWEEN SHELLS}

To verify the possibility for differentiating between shell pigments, we acquired terahertz absorbance spectra of scallopshell (in Hokkaido, Japan) powder and Meretrix lusoria shell (in Mexico) powder, and compared the absorptions with the absorption of the ground $4 \mathrm{~N}$ powder at around $3 \mathrm{THz}$. The particle size of these shell powders was less than $1 \mu \mathrm{m}$. We have verified that shell samples had different crystal structures using $\mathrm{X}$-ray diffraction analysis. Table 1 shows an example of the peak value of the diffraction angle $(2 \theta)$ ranging from $15^{\circ}$ to approximately $40^{\circ}$ using the peak search program. We could observe a calcite (104) peak at $29^{\circ}$ only in the scallop shell. The result and other peaks showed that the scallop shell was calcite, and the Meretrix lusoria shell was aragonite. Spectra of these shell powders and ground $4 \mathrm{~N}$ powder are shown in Figure 6. The $4 \mathrm{~N}$ and scallop shells, whose crystal structure is the same as calcite, had an absorbance four times as large

\begin{tabular}{|c|c|c|c|c|}
\hline & \multicolumn{2}{|c|}{ Scallop } & \multicolumn{2}{c|}{ Meretrix Lusoria } \\
\hline No. & $2 \theta\left[{ }^{\circ}\right]$ & I [cps] & $2 \theta\left[^{\circ}\right]$ & $\mathrm{I}[\mathrm{cps}]$ \\
\hline 1 & 15.580 & 138 & 26.320 & 1134 \\
\hline 2 & 23.180 & 361 & 27.300 & 594 \\
\hline 3 & 29.520 & 4025 & 32.820 & 151 \\
\hline 4 & 31.580 & 242 & 33.220 & 773 \\
\hline 5 & 39.640 & 465 & 36.180 & 598 \\
\hline
\end{tabular}

TABLE 1 Peak search data of scallop-shell powder and Meretrix lusoria shell powder from approximately $15^{\circ}$ to $40^{\circ}$ in X-ray diffraction measurements.
FIG. 5 Microscopic images of (a) $4 \mathrm{~N}$, (b) ground $4 \mathrm{~N}$ powders and (c) terahertz absorbance spectra of these powder samples. 


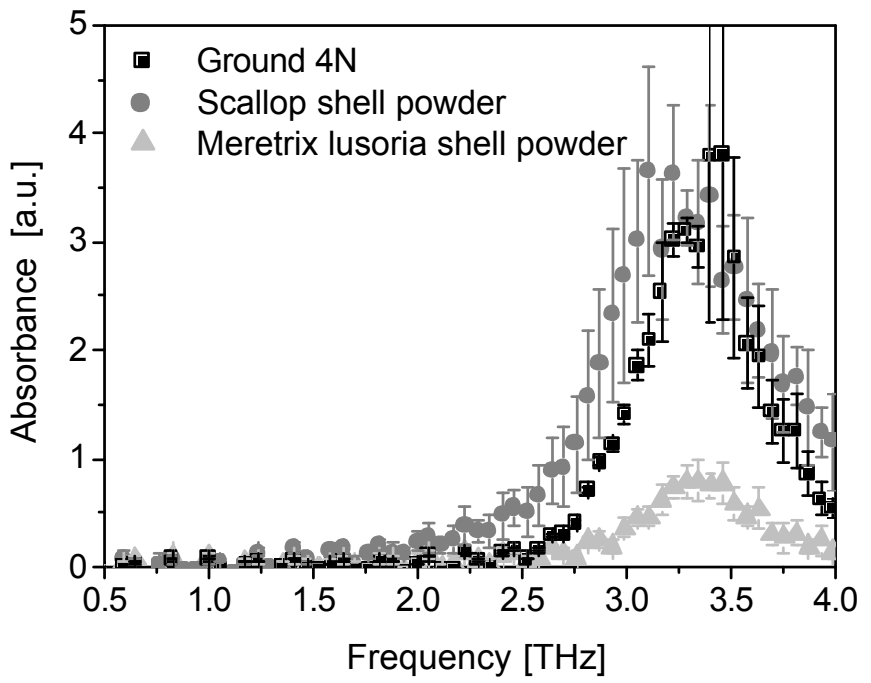

FIG. 6 Terahertz absorbance spectra of ground $4 \mathrm{~N}$ powder, scallop shell powder and Meretrix lusoria shell powder.

as the absorbance of Meretrix lusoria at around $3 \mathrm{THz}$. The absorbance peak frequencies were shifted more to the low frequency region than Meretrix lusoria [14]. Moreover, the spectral width of the scallop shell was wider than the width of $4 \mathrm{~N}$, even though the particle size of scallop shell powder was sufficiently less and more random than that of $4 \mathrm{~N}$. We conjecture that the result occurred because some molecules combined with impurities, and the scallop shell's spectrum exhibits absorption of the vibration of carbonate group shifted to the low frequency region due to inactivation. The result shows that we could detect a few impurities (such as protein) included in the shell.

From the above results, we could verify that we can differentiate between the crystal shape and the structure of $\mathrm{CaCO}_{3}$ pigment by terahertz spectroscopy, and there is potential of observing the interaction between $\mathrm{CaCO}_{3}$ and impurities from spectral width. By employing terahertz spectroscopy, we can also expect to detect a small signal, which cannot be observed in the mid-infrared region due to scattering in the powder sample.

\section{CONCLUSIONS}

The absorbance spectra of $\mathrm{CaCO}_{3}$ have been recorded by a terahertz time-domain spectrometer for the identification of a white pigment obtained from $\mathrm{CaCO}_{3}$ such as shell powder. From the results, we could verify that terahertz absorbance depended on the crystal shape, structure and the presence of impurities. We indicated that terahertz spectroscopy could work effectively in art conservation for the purpose of distin- guishing small differences in the white pigment.

\section{ACKNOWLEDGEMENTS}

The authors thank Dr. Yuichi Ogawa of Tohoku University for providing the facilities and for his useful discussions.

\section{References}

[1] A. J. Xie, C. Y. Zhang, Y. H. Shen, L. G. Qiu, P. P. Xiao, and Z. Y. Hu, "Morphologies of calcium carbonate crystallites grown from aqueous solutions containing polyethylene glycol" Cryst. Res. Technol. 4, 967 (2006).

[2] S. Ichikawa, T. Matsui, M. Sawada, M. Naruse, and Y. Matsuda, "Distinction of raw materials (shells and limestone) for calcium carbonate based on the shape of their particles II : an attempt at making a chart for distinction" JJSCCP 52, 13 (2007).

[3] C. Li, G. D. Botsaris, and D. L. Kaplan, "Selective in vitro effect of peptides on calcium carbonate crystallization" Cryst. Growth Des. 2, 387 (2002).

[4] Y. Ueno, and K. Ajito, "Analytical terahertz spectroscopy" Anal. Sci. 24, 185 (2008).

[5] M. C. Beard, G. M. Turner, and C. A. Schmuttenmaer, "Terahertz spectroscopy" J. Phys. Chem. B 106, 7146 (2002).

[6] K. Fukunaga, Y. Ogawa, S. Hayashi, and I. Hosako, "Terahertz spectroscopy for art conservation" IEICE Electron. Expr. 4, 258 (2007).

[7] A. Yamamoto, "Coulomb interactions and optically-active vibrations of calcite-type carbonates" B. Chem. Soc. Jpn. 49, 1479 (1976).

[8] E. R. Cowley, and A. K. Pant, "Lattice dynamics of calcite" Phys. Rev. B 8, 4795 (1973).

[9] H. H. Teng, and P. M. Dove, "Surface site-specific interactions of aspartate with calcite during dissolution: implications for biomineralization" Am. Mineral. 82, 878 (1997).

[10] R. Rungsawang, Y. Ueno, I. Tomita, and K. Ajito, "Angle-dependent terahertz time-domain spectroscopy of amino acid single crystals" J. Phys. Chem. B 110, 21259 (2006).

[11] A. J. Skinner, J. P. Lafemina, and H. J. F. Jansen, "Structure and bonding of calcite: a theoretical study" Am. Mineral. 79, 205 (1994).

[12] S. D. Kelly, M. G. Newville, L. Cheng, K. M. Kemner, S. R. Sutton, P. Fenter, N. C. Sturchio, and C. Spötl, "Uranyl incorporation in natural calcite" Environ. Sci. Technol. 37, 1284 (2003).

[13] Th. Posch, A. Baier, H. Mutschke, and Th. Henning, "Carbonate in space: the challenge of low-temperature data" Astrophys. J. 668, 993 (2007).

[14] A. Yamamoto, Y. Shiro, and H. Murata, "Optically-active vibrations and elastic constants of calcite and aragonite" B. Chem. Soc. Jpn. 47, 265 (1974). 